\title{
Artificial Intelligence Chatbots are New Recruiters
}

\author{
Nishad Nawaz ${ }^{1}$, Anjali Mary Gomes ${ }^{2}$ \\ Department of Business Management, College of Business Administration, Kingdom University, Riffa, Bahrain
}

\begin{abstract}
The purpose of the paper is to assess the artificial intelligence chatbots influence on recruitment process. The authors explore how chatbots offered service delivery to attract and candidates engagement in the recruitment process. The aim of the study is to identify chatbots impact across the recruitment process. The study is completely based on secondary sources like conceptual papers, peer reviewed articles, websites are used to present the current paper. The paper found that artificial intelligence chatbots are very productive tools in recruitment process and it will be helpful in preparing recruitment strategy for the Industry. Additionally, it focuses more on to resolve complex issues in the process of recruitment. Through the amalgamation of artificial intelligence recruitment process is increasing attention among the researchers still there is opportunity to explore in the field. The paper provided future research avenues in the field of chatbots and recruiters.
\end{abstract}

Keywords-Artificial intelligence; chatbots; recruitment process; candidates experiences; employer branding tool; recruitment industry

\section{INTRODUCTION}

In the new phenomenon of information technology and human resource management decades of decades have observed that, the embedded information technology and human resource management in new term as a human resource information system, digital human resource management, automation human resource, enterprise resource planning IOT (Internet of Things), data mining, [1], [2], [3], [4] and freshly artificial intelligence added to the old wine. Again, we can spot the vigor of information technology innovations in business. The latest trouble shooters (solutions) developed by technology to the complex issues of all the various functions of organizations are drawing more attention of the managers from different departments, areas and domains, not excluding the human resource department.

The term artificial intelligence (AI), commonly used for software, machines, system and computers. First time, in the era of industrial revolution, Rossum's Universal Robots (R.U.R) brought into picture by Czech Karel and it is named as ROBOT. But, in the case of artificial intelligence term has been introduced by John McCarthy (1956) appeared in the academic conference and explained the term, as he suggested artificial intelligence (AI) will contribute in future in the following specializations, like applied science, psychology, medical sciences, linguistics, biology, engineering and interdisciplinary programs.

Firstly, we need to know, why HR emerging artificial intelligence (AI), because companies want to extend their business operations to gain profit and new ventures across the globe, it is possible via new technology. Therefore, the organizations aiming to adopt automation process across the functional areas, this will minimize the time and effort of human resources, in other words artificial intelligence (AI) will replace human routine work, enforced them to generate strategies and become craft in the domain.

Artificial intelligence (AI) chatbots developed to make messages to provide assistants to the consumers for $24 / 7$, to answer all queries and acting like FB messenger, webchat, but the competitive environment enthusiastically looking for new added features in artificial intelligence (AI) chatbots to handle all the raised complex problems, therefore artificial intelligence (AI) chatbots are much demanded in chatbot market. Additionally, chatbots present organization to be data driven and pivotal in the success of the business.

The paper is structured as follows, the literature is reviewed, the purpose of the study and then discussion is presented. The paper dismisses with a conclusion and ideas for future research studies.

\section{LITERATURE REVIEW}

According to [5] recruitment process enhance quality with AI, it will assist employers to select suitable candidate with in a second to ensure whether the candidate is suitable or not. AI has constantly developed over the time to provide deeper insights. This will ensure the organizations not only hire, the right candidate for the organization but also with right skill. [6] main aim of the study is to explain digital technologies influence HR organization. This paper throws light on how digital technologies have reconfigured the HR organization as well as digital technologies transformation and support to the organizational effectiveness, talent strategy execution, succession planning, monitoring operations, transactional case monitoring, recruitment process (robotics automation, artificial intelligence (chatbots), [7], workforce planning, analytics, employee banding management, rewards and engagement, employee relations and effectiveness of organization and finally concluded that digital technology forcing $\mathrm{HR}$ process and organization structure into the transformation and [8] through AI can identify right talent leaders, they may easily procure deep insights of training needs, how to cradle time consuming in screening the resumes, unbiased candidate screening and helpful in analyzing personality traits and finally concluded that conduct onboarding via chatbots, this will enhance workforce experience. AI is significantly improving in HR functions, especially in performance management. Traditionally HR managers are evaluating employees performance once in a year, but AI-driven performance management removing unwanted delay in measurement of performance via real time points, face to face communication, chatbots and these technologies providing daily performance, this will prioritize retention across HR [9]. 
Artificial intelligence understands the human language and not only command but learning from human conversion, transforming like intelligent agent, chatbot is a computer program the conversations through an auditory or methods of textual via natural language processing (NLP), natural language understanding (NLU) and natural language generation (NLG) for interacting humans like ALICE. [10].

An automated mediator stimulate job-seeker to ask questions directly with recruiter about the salary, incentives, leave facilities, FAQ, workforce diversity, complex queries and other related questions [11]. Another piece of work done in this direction chatbots eliminate the routine work in the recruitment process, the RobRecruiters chatbots are automating end to end recruitment process and doing regular process of attendance tracking, goal tracking, reviews of performance, surveys related employees, balance leaves and other activities, enables the HR managers and HR team to move towards success to place organization in top in digitalized era. [12].

(Adams, 2018) chatbots are transforming and assisting in recruitment process to know candidate experience, for effective communication between candidate and recruiter, questions and answers, in identification of qualified candidate and to schedule conversation and finally chatbots taking all requirements from the candidate before his/her entry into the organization. The modern recruiters having more additional responsibilities to have strategies to meet the assigned business goals, keep tracking of competitors, keeping realistic challenges via benchmark, tracking of employees' satisfaction level in all the stages, for this chatbots are useful, because there is a daily conversation database, this will be useful to resolve complex issues Joshi (2019).

The artificial intelligence empowered chatbots to execute human conversation in messaging, the unique way of using words, shorthand, emotions, [13] at the end it will work on the basis of natural language to support conversation process [14], the studies are rare in the field of recruitment process. The authors claim that our understanding of artificial intelligence in recruitment process would benefit more intensive by across all levels of recruitment process. In order to develop better understanding of the recruitment process in human resources organizations in general, at the same time to have effectiveness in different areas of recruitment. Therefore, the authors have proposed the study of artificial intelligence chatbots influence in recruitment process.

\section{Methodology}

The present study is completely based on literature reviews. The collected literature provided basic understanding of artificial intelligence chatbots and its flow in the recruitment process. To survive this purpose author's selected secondary data such as journals, websites and reports to develop the entire paper.

Additionally, the authors have used library database such as Scopus, ProQuest, EBSCO, Science Direct, Emerald, Elsevier, Taylor and Francis, Springer and Wiley inderscience. The main keywords used for the research include, artificial intelligence, chatbots, recruitment, recruitment process and
Internet search engines through Google, Google scholar were utilized to identify and access the relevant working papers, reports, blogs and presentations were used to ensure comprehensive coverage of the literature.

\section{RESULTS}

The results identified that there is an increase in the technology development in human resource management, especially in the recruitment process that will have more influence in the future. In past years companies used various technologies for the recruitment such as social media, employee portals, job portals, internal and external networks, social networking, peer referral, emails, mobile messages, cell phone communications are used to attract star performers, best talents for their teams to perform better in the organisation.

In the present transparent digital era, the recruiters, are more experienced because the candidates are more tech-savvy in the mobile environment, and it reinforces the employment brand, an organization employment brand is extremely important, it will attract best performers into their talent pool. Therefore, organizations always want to manage their employment brand to pull candidate towards them.

There are many new implications, solutions and innovative ideas around cognitive technologies, for instance, artificial intelligence, natural language processing, natural language understanding, natural language generation, machine learning, predictive algorithms and robotics process automation, after introduction of Olivia, the chatbots become very popular in the recruitment market and it is providing the guidelines to the candidates, answer their questions. Moreover, facilitating sequence questions to the applicant to answer.

The above technologies adopt smart methods for collecting data of the candidate to make progress in various techniques [15] to identify possible candidate to apply, responding to the unsuccessful candidate, screening the candidate, in sending job offer, and bringing selected candidate into company [16] in other way connecting \& collecting all the information of employee into single database and it reveals a new insights for better candidate profile to hire and improve the effectiveness of the recruitment process [17].

Against this reflection, the aim is to study AI chatbots impact in the recruitment process. More precisely, the present study attempts, AI chatbots, significance across the recruitment process function. These are discussed below.

\section{DISCUSSION}

\section{A. Simplify the First Stage}

The present chatbots can accomplish a lot. They can interpret resumes and request elucidations. Interacting with applicants one to one, instant messaging conversations on platforms like Facebook Messenger and text messages, chatbots can get some information about the applicant's experience, answer usual inquiries, and gather a wide range of data and request for a human selection representative to analyze.

Once the application is received screening these applications is an incredibly tedious procedure. Recruiters 
generally affirm which applicants are appropriate amid the first round of pre-screening calls; while we do know this works, this can be a long procedure which needs revising. Chatbots are an extraordinary arrangement which can make this phase of the evaluation unmistakably increasingly effective! By conveying a text message to every potential applicant that prompts a progression of short, precharacterized questions, the appropriate applicants can be effectively be sifted through from the unsatisfactory.

Recruiters' can convey several texts in a few minutes and get reply speedily, as compared to the days or even weeks that telephone calls and messages may take.

\section{B. Get the Right Data at the Right Time of the Right Candidate}

All organizations dream of updating candidates' database every day, replying to clients faster as well as creating a long term relationship with the candidates. Its not a dream anymore, it is now possible with chatbot. Companies can deploy a chatbot connected to the database to regularly update it. Companies can check their database information which is a key influence. With individual and automated discussions companies can refresh applicants' accessibility, their present position, their mobility or even a new certification. Companies can add value to their database by deploying a chatbot within the preferred audience which can add value to the database.

\section{Qualifying Candidates}

Chatbots can perform an excellent job of filtering out the good from the bad by asking questions to the applicants related to skills, qualifications, and past experiences which can be otherwise a tedious and time consuming task for the recruiters. It can then effectively rank and qualify a whole group of candidates in terms with the required criteria of the organization.

With all the extensive administrative tasks that come with hiring a candidate, Chatbots can take off a lot of the load by setting up inevitable calls and scheduling meetings keeping in mind both the parties. Apparently, these stages of the process require the human but chatbot ensures all requirements are addressed before humans take over.

\section{Get more Qualified Appliers into Job Offers}

Companies are aggressively forwarding emails to their database to get more job applicants however the achievement rate isn't as high as anticipated. With a chatbot on messaging apps companies can draw in the applicants database and push them towards the right job at the opportune time. Applicants can apply without any difficulty through the chatbot.

There is no signing in required to go on a website nor have they to go through tedious application process. The job applicants will receive job offers on their messaging apps rather than receiving it through emails. At that point, they can apply for them without changing to another application or site.

\section{E. Increased Numbers of Applications}

Due to the impact of social media, facebook recruitment via facebook groups, posts are becoming extremely popular in attracting new candidates. However, the problem is to persuade the applicant to click on the company's career page and submit their application as well. The solution is very simple in the chatbots. By using an automated facebook messenger recruitment chatbot, any potential applicant could be prompted to opt-in for job alerts and provide them with their facebook profile information, as well as showing them job openings, information about the process of application or even videos about working at the company. The possibility of submitting the application by the candidate increases many folds if they are engaged right from the point of initial interest as they've already had contact and established a rapport with the company

The shocking fact is that as per Jobvite Recruiting Funnel 2017 only 8.52 per centage of visitors to a career site literally complete their application. Which means more than 91 per centage of potential candidates just leaves the site without leaving any information for the company, hence even if the company wants to contact them in the future they cannot due to lack of information. This can be a very big problem to companies trying to attract talented and skilled people for their job vacancies. To tackle this issue a chatbot can help by engaging candidates through a messenger, replying to any questions regarding any misgivings they have which might daunt them from submitting any application, it can also give reminder to candidates to complete all the information in case it is insufficient.

\section{F. Question and Answer (FQA)}

Before applying the applicants needs to know about the job, the company and various requirements and it is extremely frustrating if a candidate has to look for all these answers on an FAQ page. This can be completely changed with implementation of chatbots. Companies can use chatbots to answer FAQ by recognizing keywords mentioned by the applicant. It's crucial to make the answers understandable and informative which will enable the applicant to acquire knowledge and clear understanding promptly leading to applicant's satisfaction.

It is less intimidating communicating to a chatbot where an applicant can be just themselves and stay calm and composed. Chatbots can respond to the applicants in a user-friendly way and if the answer is not known it will refer the applicant to the right person to create a contented experience. This is the age of data driven decisions hence a chatbots could be linked to a platform which can gather important data. This type of platform will enable the company to tag how many times a particular question has been asked and what candidates want and are looking for. Chatbots are great assistance to recruiters with their prompt replies and instant availability.

\section{G. Responding to the Unsuccessful}

According to Eyal Grayevsky, Mya "applicants who never get to hear back after submitting their applications from the recruiter are approximately 85 per centage" this results in poor applicant experience and a wrong impression of the company. This can also lead to loosing potential candidates which might be a critical issue, as they will not be motivated to reapply in the same company, who might be better suited for another position in that company in the future. 
To remain competitive companies must attract the best talent and skilled professionals. The companies can be successful in this only if they are seen as someone who treats people with respect. With numerous numbers of applications for one job position it is practically not possible for recruiters to personally reject everyone, hence it makes the chatbots the most suitable option. Chatbots can promptly respond to the applicants once a decision is taken. The applicants acknowledge this kind of swiftness and at the same time they don't develop any negative feeling towards the organization as they don't go through the anxiety of waiting time.

\section{H. Screening Candidates Application}

Chatbots can initiate a conversation with the applicants once they apply on a company's job site. Chatbots may ask many questions while communicating with them. The questions can range from asking about work experience, previously where they have worked, their areas of interest and so on. When this process is over the chatbots assess the applicant for relevancy of the unfilled position. The decision is taken by the recruitment chatbot based on the conversation that took place, resume details and assessing the job requirement if the candidate is best bit for the job.

\section{Assess Candidates' Recruitment Experience}

Companies must be aware about how the applicants feel regarding their recruiting experience. Its important to differentiate from the competitors which is possible by getting feedback and this way a strong relationship can be built. To enhance the retention rate of the company its important to ask the applicants about how they feel. The chatbots can assist in checking candidate's feedback after interviews and get deep insights about how companies create a bond with people within the talents pool and useful in recruitment strategy as well.

\section{J. Interview Scheduling of the Candidates}

Scheduling the interview with the candidate is another time-consuming task. Intellectual chatbots are capable of accessing the calendar of the recruiters to check if they are available and then schedule the date and time for the relevant candidate. In today's time it's not very much effective to just make calls to the candidate as most of them don't answer to unrecognized phone numbers. Also, it might be bothersome to call the candidate when they are working with their current company or they have to request for a convenient time for both the parties. This whole process could be very time consuming. But for a chatbot this task will not be tedious, and they are great in this type of repetitive tasks.

\section{K. Enhance Candidates On-Boarding}

The very first step for a candidate is on-boarding in an organization which is also a long and key process. To smoothen the process the organizations can deploy a chatbot to deliver significant information at the right time to the newly recruited employees.

\section{Candidate Experience}

It's nothing astonishing that one will need to get a chatbot engaged with this piece of the procedure. With a new job opening numerous numbers of applicants will be pulled in a high volume, it very well may be a major errand for a human to deal with. Proficiently that is, chatbot can intercede with faster reply and speed up the procedure.

To emerge of the commotion, recruiters ought to possibly change their recruitment strategy and make it more applicant driven. The time that applicants take to send resumes and recruiters hit them up ought to be short. Chatbots can get this going. Chatbots can return to the applicants quickly, making the applicants as well as the recruiters contented on that front. With a correct approach companies can make the job search experience where conversation is concerned a more natural process.

In the present day's marketing recruitment the experience of the applicants is becoming extremely important. The applicants' journey must be effortless, straightforward, and inviting and this must be ensured by the talent acquisition leaders. Recruitment chatbots can connect with applicants in a conversational trade as well as answer recruiting FAQs, a boundary that prevents numerous applicants from applying. With recruiting web chat arrangement like career chat, applicants can study the organization and draw in recruiters in live agent modes and computerized modes.

\section{Candidate Experience Feedback}

Huge number of applicants have poor encounters while presenting their applications and resumes on the web. As a rule, they don't get advised about whether an organization they have connected for has gotten their documents. This vulnerability combined with the distress of sitting tight for input makes a negative impact on an organization's validity.

Through the incorporation of selecting artificial intelligence, many applicants can be obliged promptly and advised with the outcomes of their interview once it's finished. This brings down their nervousness and encourages them proceed onward to discovering openings that are more appropriate for them. Organizations that make HR bots are looking for better approaches to improve their process to fulfill the requirements of clients around the world.

Job pal perceives the requirement for organizations to begin connecting with applicants the minute they apply for work/ job. With that, they have fabricated AI-controlled chatbots to mechanize the correspondence among employees and applicants accordingly accelerating the procuring procedure.

\section{N. Notation Feedback}

Structured inquiries that accompany predefined answers will aid in real-time feedback. Instead of using forms the applicants can have the privilege of dynamic interactive conversational interface to share their feedback. In the point of improvement, Questions from the employers can be put forward to candidates such as, "where do you think we need to improve" to get an understanding of areas of improvement. The feedback of the applicants could be shared with the recruiters to initiate necessary actions and filter the process to best fit the human capital needs. Using these observations the companies can make sure of providing an engaging experience to candidates and help HRs in eradicating human bias and the possibility for any error. 


\section{O. Building Rapport}

Correspondence is significant amid the recruitment lifecycle. That is for both the job applicant and recruiter. The informing perspective accessible with chatbots makes it a simple and natural methodology. The chatbots are fueled through guidelines. They're savvy. A chatbot will pose decision inquiries and ensure the discussion is appropriate. Continually recording subtleties prepared for the recruiter to meet the job applicant.

For instance, Mya is an excellent case of a viable chatbot. Planned just for enrollment, it talks and draws in with job applicants through a messaging app. It records every one of the information and answers questions asked by the job applicant. Along these lines, it builds profitability as you can qualify an expansive number of job applicants.

\section{P. Employer Branding Tool}

Employer branding helps the company to be outstanding if on the career page of the company have a chatbot. To increase the retention rate of the employees it's very important to engage the right candidates. It's unfortunate and sad that employer branding is often extravagant and time-consuming because it includes building content such as article, blog posts, employee interviews, videos, etc. It continues with marketing all these content to the pool of initial candidates.

Though a lot of organizations are excellent in building employer branding content and posting that content on their career site but unfortunately not many of the candidates are seeing it, but with chatbots it is very much possible to send automated messages to the current subscribers, with the most recent blog posts, videos, etc. all-over multiple messaging platforms.

By giving a personality to the recruiting chatbot some of the companies are taking their branding to the next level. The companies also make sure that the culture and values of the organizations are well aligned to the chatbot and its interactions and promote the employer brand by making sure there is regularity in messaging during the recruitment process.

\section{CONCLUSION}

Indeed, artificial intelligence has it existence in recruitment process and smartly work like human brain in various complex situations. Digital era gains more attention and importance in automating recruitment process when compare to traditional system of recruitment. Artificial intelligence provides smooth process by conducting screening of $\mathrm{CV}$, responding with automated message. Adoption of artificial intelligence has helped in increasing to build momentum, by reducing tedious routine work of recruiters through AI chatbots.

This technology has been successful in simplifying the work and collect related information in candidates experience, building relationship, to answer questions, identification of right candidates, on-boarding, increasing of applications, scheduling interviews and so on will be align with recruiters to have smooth functioning of process to make business success. The study has some limitations. The aim of the study is to know chatbots influence on recruitment process, how it is improving performance of the recruiters. Additionally, it was not studied why recruitment industry is very keen in adopting chatbots in recruitment in the present study.

\section{VII.FURTHER RESEARCH DIRECTIONS}

The papers discussed available facets of technology used in recruitment industry for recruitment process. The present study presented fundamental base for future research work in field of AI chatbots and recruitment process. In the future the researchers can take privilege to add to new literature to above discussed topic. The researchers can conduct empirical studies with different perspective. The enthusiastic researchers can take comparative studies before introduction AI chatbots and after in the recruitment process, industry wise, HR designation wise also possible to study. The authors predicting that present study act like "food for brain" for researchers in the field of artificial intelligence.

\section{REFERENCES}

[1] Ernst and Young, "The new age: artificial intelligence for human resource opportunities and functions," USA, 2018.

[2] D. L. Stone, D. L. Deadrick, K. M. Lukaszewski, and R. Johnson, "The influence of technology on the future of human resource management," Hum. Resour. Manag. Rev., vol. 25, no. 2, pp. 216-231, Jun. 2015.

[3] T. Bondarouk, E. Parry, and E. Furtmueller, "Electronic HRM: four decades of research on adoption and consequences," Int. J. Hum. Resour. Manag., vol. 28, no. 1, pp. 98-131, Jan. 2017.

[4] N. Nawaz and A. M. Gomes, "Human resource information system: a review of previous studies," J. Manag. Res., vol. 9, no.3, p.92, Jul. 2017.

[5] N. Nawaz, "A comprehensive literature review of the digital HR research filed," Information Knowl. Manag., vol.7, no.4,pp.15-20, 2017.

[6] J. Gikopoulos, "Alongside, not against: balancing man with machine in the HR function," Strateg. HR Rev., vol.18, no. 2, pp. 56-61, Apr. 2019.

[7] A. DiRomualdo, D. El-Khoury, and F. Girimonte, "HR in the digital age: how digital technology will change HR's organization structure, processes and roles,"Strateg. HR Rev,vol.17,no.5,pp.234-242,Oct. 2018.

[8] N. Nawaz, "Robotic process automation for recruitment process," Int. J. Machanical Eng. Technol., vol. 10, no. 04, pp. 88-91, 2019.

[9] E. He, "Can artificial intelligence make work more human?," Strateg. HR Rev., vol. 17, no. 5, pp. 263-264, Nov. 2018.

[10] B. Buck and J. Morrow, "AI, performance management and engagement: keeping your best their best," Strateg. HR Rev., vol. 17, no. 5, pp. 261-262, Nov. 2018.

[11] P. Nikhila1, G. Jyothi2, K. Mounika3, M. C. Kishor, K. Reddy, and R. Murthy, "Chatbots using artificial intelligence," J. Appl. Sci. Comput., vol. 6, no. 2, pp. 103-115, 2019.

[12] K. Kuksenok and N. Praß, "Transparency in maintenance of recruitment chatbots," in Where is the Human? Bridging the Gap Between AI and HCI Workshop , 2019, pp. 1-4.

[13] B. Sheth, "Chat bots are the new HR managers," Strateg. HR Rev., vol. 17, no. 3, pp. 162-163, Apr. 2018.

[14] A. Følstad and P. B. Brandtzæg, "Chatbots and the new world of HCI," Interactions, vol. 24, no. 4, pp. 38-42, 2017.

[15] J. Hill, W. Randolph Ford, and I. G. Farreras, "Real conversations with artificial intelligence: a comparison between human-human online conversations and human-chatbot conversations," Comput. Human Behav., vol. 49, pp. 245-250, 2015.

[16] E. Parry and V. Battista, "The impact of emerging technologies on work: a review of the evidence and implications for the human resource function,” Emerald Open Res., vol. 1, p. 5, Jan. 2019.

[17] P. Cappelli, P. Tambe, and V. Yakubovich, "Artificial intelligence in human resources management: challenges and a path forward," SSRN Electron. J., Nov. 2018. 